\title{
A multi-objective model for locating distribution centers in a supply chain network considering risk and inventory decisions
}

\author{
Sara Gharegozloo Hamedani ${ }^{\mathbf{a}^{*}}$, Mohammad Saeed Jabalameli ${ }^{\mathrm{a}}$ and Ali Bozorgi-Amiri ${ }^{\mathrm{b}}$
}

${ }^{a}$ Department of industrial engineering, Iran University of Science and technology, Tehran, Iran

${ }^{b}$ Department of industrial engineering, branch of technical faculties, Tehran University, Tehran, Iran

C H R O N I C L E

Article history:

Received December2, 2012

Received in revised format

10March 2013

Accepted 14March 2013

Available online

March14 2013

Keywords:

Facility location

Location-inventory

Risk management

Supply chain network design

Multi-objective optimization

\section{A B S T R A C T}

\begin{abstract}
This paper presents a multi-objective location problem in a three level supply chain network under uncertain environment considering inventory decisions. The proposed model of this paper considers uncertainty for different parameters including procurement, transportation costs, supply, demand and the capacity of various facilities. The proposed model presents a robust optimization model, which specifies locations of distribution centers to be opened, inventory control parameters (r, Q), and allocation of supply chain components, concurrently. The resulted mixed-integer nonlinear programming minimizes the expected total cost of such a supply chain network comprising location, procurement, transportation, holding, ordering, and shortage costs. The model also minimizes the variability of the total cost of relief chain and minimizes the financial risk or the probability of not meeting a certain budget. We use the $\varepsilon$ constraint method, which is a multi-objective technique with implicit trade-off information given, to solve the problem and using a couple of numerical instances, we examine the performance of the proposed approach.
\end{abstract}

\section{Introduction}

In today's business environment and trade globalization, most companies, customers and suppliers are in connection with each other like a chain and the key success is to have an efficient supply chain management to decline the cost of supply chain and to enforce its performance. Supply chain contains a network of suppliers, manufacturers, warehouses, distributors and customers, to convert raw material to final products, deliver them to customers to meet their demands aiming to minimize (maximize) cost (profit) of the whole chain. Strategic design of supply chain network is one of the most important elements of supply chain and it influences on tactical and operational decisions. One of the major decisions for designing a supply chain network is to locate various parts, which require high investments. In a supply chain, a company seeks to locate facilities such as plants, distribution

Corresponding author. Tel:+989113390727

E-mail: gharegozloo_sara@ind.iust.ac.ir (S. GharegozlooHamedani)

(C) 2013 Growing Science Ltd. All rights reserved.

doi: $10.5267 / \mathrm{j} . \mathrm{ms} 1.2013 .03 .020$ 
centers, retailers, which could maximize (minimize) total profit (cost), and for a long-term planning, it is crucial to consider both operational and tactical decisions, simultaneously (Shen et al., 2003, Shen and Qi ,2007,Sourirajan et al., 2007). This issue has caused developing of integrated inventory location models in recent years to integrate tactical and strategic decisions and to develop inventorylocation models, which could benefit from risk pooling effects (Daskin et al., 2002; Shen et al., 2003, Eppen 1979; Berman et al., 2012). In addition, the significance of uncertainty as an inevitable part of continuously changing business, has motivated a number of researchers to address stochastic optimization in supply chain network design involving locating facilities, distribution of commodities by probabilistic scenarios representing uncertainty.

Melo et al. (2009) performed a comprehensive review on the literature of supply chain management for integrating different types of decisions during strategic decision making specially locationinventory decisions. Nozick and Turnquist (1998) studied how to include inventory costs (contingency reserve) in the classic problem of locating facilities of the plant. Erlebacher and Meller (2000) had a broader view and studied a nonlinear integer inventory-location model by considering fixed costs, transportation and inventory costs. Shen et al. (2003) studied facility location problem in which facilities manage their inventory through policy of (r,Q). Daskin, et al. (2002) presented an efficient solution based on Lagrangian relaxation approach, which solves the model faster than the method of Shen et al. (2003) does. Teo and Shu (2004) studied the problem of logistic network considering inventory costs for multilevel locations of inventory storage. Romeijn et al. (2007) studied the previous model by adding term of contingency reserve (considering potential demand). Snyder et al. (2007) studied potential state of this model but there are few models in literature, considering uncertainty in other parameters, except demand. Snyder et al. (2007) used scenario approach to include uncertainty on parameters. Rawls and Turnquist (2010), Jia et al. (2007) presented an uncapacitated facility location model to locate emergency service facilities in the event of a large-scale emergency. Chang et al. (2007) modeled locating and distributing rescue resources in a flood emergency under possible flood scenarios using two-stage stochastic programming.

Moreover, Qi et al. (2010) studied the effect of disorder in facilities for two-leveled supply chain, a supplier and several retailers, and tried to locate retailers optimally and allocate customers to them. Chen et al. (2011) studied a reliable inventory-location model to optimize facility location decisions, allocation of customers and management of inventory in case distribution centers are at risk of disorder. Üster et al. (2008) studied single facility location, which includes location decisions, inventory completion (reordering periods), ordering costs, transportation and holding of inventory. Park et al. (2010) proposed a design model for three-level network by considering the contingency reserve. Tancrez et al. (2012) considered inventory-location problem for three-level supply chain in which distribution decisions of distribution centers, allocation and amount of the transported commodity were made altogether. Berman et al. (2012) studied an inventory-location model in which distribution centers had inventory control policy (R,S). Tsao et al. (2012) studied the allocationinventory-location integrated problem for designing a distribution network with several local distribution centers and retailers. For more details, interested readers are referred to Gharegozloo et al. (2013). In real supply chain systems, if the support facilities are considered for customers, reliability of supply chain and its general performance would be considerably improved to handle a more realistic situation, in this paper, we consider back up DCs.

In addition to conquer the disadvantages of traditional, stochastic supply chain design approaches namely minimizing (maximizing) cost (profit)as a single objective, minimizing the risk reflected by variance of total cost and not considering financial risk, etc.(Azaron et al. 2008), robust stochastic programming approach is developed to design the uncertain supply chain using discrete scenariosbased approach, each with a certain probability of occurrence. The model aims to determine:

- Number of DCs to be opened,

- The location of these DCs, 
- The amount of inventory should be kept,

- The items need to be delivered from each supplier to each DC and each DC to a customer.

For the proposed model of this paper, which is an extension of another work published earlier by authors, Gharegozloo et al., 2013, the first objective function is to minimize the expected total cost of supply chain, including procurement, transportation, holding, ordering and shortage cost. To develop a robust stochastic model, two additional objective functions are added into the model. The first additional objective function is to minimize the variance of the total cost of supply chain. The variance of the total cost should be considered in the model, because concentrating only on the expected total cost, may lead to sub-optimal solution if the total cost significantly alters because of randomness (Mulvey et al. 1995). In practice, the interpretation of the total cost variance is hard, so, adding a new objective function capturing risk, is necessary (Azaron et al., 2008). The purpose of the objective function is to minimize the financial risk, which is defined as the probability of not meeting a certain cost level. Actually, the concepts of variance and financial risk have been considered in other areas, but to the best of our knowledge, it is the first time they are considered all together in a multi-objective scheme to model robust supply chain under uncertainty considering inventory decisions. The main contributions of this paper, which differentiate this paper from the existing ones in the related literature; can be summarized as follows:

- We introduce a comprehensive new robust optimization model to deal with the supply chain and also integrates the strategic planning, such as the location of DC, with tactical decisions, i.e., inventory policy to avoid the separated decision making processes between strategic and tactical levels.

- Our robust formulation not only takes into account the expected total cost of supply chain but also considers the risk reflected by the variance of the total cost.

- Offering a mathematical programming model to handle different sources of uncertainties using a scenario-based approach, attempting to capture uncertainty by representing it in terms of a number of discrete scenarios.

- Our model considers capacity constraint on suppliers and DCs and allows for multiple capacity levels to the DCs.

We formulate the distribution centers location problem as a multi-objective robust stochastic mixedinteger nonlinear programming problem. The results multi-objective problem, after linearization, is solved using $\varepsilon$-constraint technique where there are conflicting objectives, simultaneously. The results of the model suggest a set of Pareto optimal solutions and create an opportunity for us to find the best supply chain configuration according to his/her viewpoint.

The rest of this paper is arranged as fallows. In section 2, a brief description of robust optimization is presented. In section 3, the given problem is formulated as a robust optimization model. In section 4, a Solving procedure based on epsilon constraint technique is explained. Section 5 presents the numerical examples. Conclusion and future research of this study is presented in section 6 .

\section{Robust optimization}

Robust programming, introduced by Mulvey et al. (1995), is an improved stochastic programming to deal with the preferred risk aversion of decision makers, which was not possible to use in routine stochastic programming (Bozorgi Amiri et al., 2012; Azaron et al., 2008) to locate distribution facilities in a three levels supply chain network under uncertainty. In this method, the variability term was supplemented to the main objective function by a related weighting parameter to show the tolerance of modelers' risk. In the remaining, a concise description of robust optimization is presented. Let $x$ be first stage (design) variable vector and $y_{s}$ be the second stage control variable vector. Let $M, N, O$ be matrices of parameter and $p, q$ be vectors of parameters. Let $M, p$ be certain 
parameters and $N, O, q$ be uncertain ones. Let $S$ be the set of scenarios to model uncertainty with associated probability of occurrence of each scenario $\left(p_{s}\right): S=\{1,2, \ldots, s\}$ and $\sum p_{s}=1$ so we have $N_{s}, O_{s}$, $q_{s}$. The possible infeasibility of model is presented by $\delta_{s}$. (if model is feasible 0 , otherwise a positive value is calculated by Eq. (3) model formulation is as follows (Bozorgi-Amiri et al. 2012; Gharegozloo et al. 2013):

$\min \sigma\left(\mathrm{x}, \mathrm{y}_{1}, \ldots, \mathrm{y}_{\mathrm{s}}\right)+\omega \mathrm{p}\left(\delta_{1}, \ldots, \delta_{s}\right)$

subject to:

$\mathrm{Mx}=\mathrm{p}$,

$\mathrm{N}_{\mathrm{s}} \mathrm{x}+\mathrm{O}_{\mathrm{s}} \mathrm{y}_{\mathrm{s}}+\delta_{s}=q_{s} \quad \forall \mathrm{s} \in \mathrm{S}$

$\mathrm{x}, \mathrm{y}_{\mathrm{s}}, \delta_{s} \geq 0$.

The first term in Eq. (1) shows the solution robustness, which seeks for less cost and risk aversion degree. The second term indicates the model robustness, which gives penalty solutions by unmet demands or exceeding from each physical constraint. $\omega$ is a weight measuring the trades-off between the first and the second term of Eq. (1). According to Bozorgi-amiri et al. (2012), we use $v_{\mathrm{s}}=\mathrm{z}\left(\mathrm{x}, \mathrm{y}_{\mathrm{s}}\right)$ as the main objective function under scenario $s$. The solution is a high-risk decision when the variance of $v_{\mathrm{s}}$ is high. Mulvey et al. (1995) used a quadratic form of variance, which is nonlinear and complicated. To handle this difficulty, here we use an absolute deviation as Yu and Li (2000) proposed, which is as follows,

$\sigma(o)=\sum_{s \in S} p_{s} v_{s}+\lambda \sum_{s \in S} p_{s}\left|v_{s}-\sum_{s^{\prime} \in S} p_{s^{\prime}} v_{s^{\prime}}\right|$,

where $\lambda$ is the weight of the less sensitive-solution to data changing in all scenarios. For minimizing the Eq. (5), Yu and Li (2000) presented an effective method, which is modeled as follows,

$\min \sum_{s \in S} p_{s} v_{s}+\lambda \sum_{s \in S} p_{s}\left[v_{s}-\sum_{s^{\prime} \in S} p_{s^{\prime}} v_{s^{\prime}}+2 \theta_{s}\right]$

subject to:

$v_{s}-\sum_{s \in S} p_{s} v_{s}+\theta_{s} \geq 0 \quad \forall \mathrm{s} \in \mathrm{S}$

$\theta_{S} \geq 0 \quad \forall \mathrm{s} \in \mathrm{S}$

If $v_{\mathrm{s}}$ is greater than $\sum p_{s} v_{s}, \theta_{s}$ is equal to 0 , otherwise $\theta_{s}=\sum p_{s} v_{s^{-}} v_{s}$. In this study, we use Yu and Li's method but as the expected value of costs and their variance are in contrast, we form a two-objective model, which separates the two presented terms in Eq. (6) in order to enhance the model efficiency and find Pareto solutions. This provides a good condition for decision makers to make decision according to their preference.

\section{Problem description}

In this section, an integrated multi-objective robust facility location model, by considering risk and inventory issues, is presented to design a supply chain network including suppliers, distribution centers and customers as integer nonlinear programming in case of uncertainty, which integrates location, inventory and allocation decisions. The presented model's hypotheses are as follows (Gharegozloo et al. 2013):

\subsection{Assumptions}

1- More than one product can be supplied in the chain, each one of the products has different 
volume, procurement, shortage, holding, ordering, and transportation cost.

2- Capacity of the suppliers and distribution centers is subject to uncertainty due to events such as fire, earthquake, etc.

3- There is uncertainty in parameters such as demand, supply, purchase price and costs and some discrete scenarios belonging to the set of possible scenarios $\mathrm{S}$ are used for showing this problem.

4- There are some candidate points for establishment of distribution centers, which are selected for each location's fixed cost.

5- Each distribution center can be constructed only in one of the available sizes (small, medium and large).

6- Each distribution center can be supplied from suppliers and other distribution centers (logistic cover) if possible.

7- There is no limitation of single-facility supply for customers and distribution centers.

8- Inventory is kept only in distribution centers. In this case, this inventory is fined.

9- Remaining inventory holding cost is different depending on whether the remaining inventory is related to supplier or logistic distribution center.

10- The package shortage cost differs depending on whether shortage results from failure to select customer for providing services because there is uneconomical or insufficient distribution center to provide services while the model is as the lost sale.

11-In this model, there is inventory ordering policy (r,Q) and EOQ approximation approach has been used to determine its parameters based on the work of Axsater (2006). It has also risk pooling property.

12- A certain cost level or budget is considered so that the total cost must not exceed from this level.

this model's firs objective function is to design a distribution network to specify location and number of distribution centers, inventory order amount in each one of the distribution centers, the allocation of customers to distribution centers as well as distribution centers to suppliers by aiming at minimizing the expected value of costs and variance of these costs. Variables of the first stage (design) and the related fixed location cost were final but variables of the second stage (control) and its related parameters such as demand, supply, etc. are assumed uncertain. Uncertainty is captured by some specific discrete scenarios. In the remaining, the symbols relating to this problem are presented.

\subsection{Indices}

$I: \quad$ set of suppliers

$J: \quad$ set of distribution centers

$K$ : set of customers

$L: \quad$ set of the assumed sizes for distribution center (small, large, medium)

$S: \quad$ set of possible scenarios

$C$ : set of all demanded commodity

\subsection{Deterministic Parameters}

$f_{j l}: \quad \quad \quad$ Fixed cost of opening distribution center $j$ with size $l$,

$F_{j c}$ : $\quad$ Fixed ordering cost of any distribution center $j$ for each commodity $c$,

$P_{s}: \quad$ Probability of occurrence of scenario $s$

$\pi_{k c}$ : Shortage cost in distribution centers for one unit of commodity $C$ resulting from demand of customer $k$ (the penalty of un-met demand of assigned customer to a DC due to uncertainty of supplier),

$\pi_{k c}^{\prime}$ : Shortage cost resulting from failure to allocate some of the customer's demand $\mathrm{k}$ for each commodity unit $C$ (lost sale),

$v_{\mathrm{c}}$ : $\quad$ Per unit required space for each commodity $C$,

$S_{i c}$ : $\quad$ Amount of commodity $c$ which supplier $i$ can supply,

Cap ${ }_{l}$ : Different type of opened DCs' capacities (in cubic meters), 
$h_{j c}$ : Holding cost of a commodity unit $c$ in distribution center $j$ (in case it receives commodity only from suppliers),

$h_{j c}^{\prime}$ : Holding cost of a commodity unit $c$ in distribution center $j$ (in case it receives commodity from supporting distribution centers in addition to suppliers),

$\alpha: \quad 1$ in case the support is used and 0, otherwise,

$M$ : A large number,

$B U D$ : A pre-determined budget.

\subsection{Nondeterministic Parameters}

$q_{j s}: \quad$ A percentage of capacity $j$, which remains active under scenario $s$,

$q_{i s}: \quad$ A percentage of capacity $i$, which remains active under scenario $s$,

$\varphi_{i c s}: \quad$ Purchase cost of a commodity unit $c$ from supplier $i$ under scenario $s$,

$\varphi_{j^{\prime} c s}: \quad$ Purchase cost of a commodity unit $c$ from supplier $j$ under scenario $s$,

$C_{i j c s}$ : Transportation cost from supplier $i$ to distributor $j$ for each commodity unit $c$ under scenario $S$,

$C_{j^{\prime} j c s}$ : $\quad$ Transportation cost from logistic distributor $j^{\prime}$ to distributor $j$ for each commodity unit $c$ under scenario $s$,

$C_{j k c s}$ : Transportation cost from distributor $j$ to customer $k$ for each commodity unit $c$ under scenario $s$,

$\mathrm{d}_{\mathrm{kcs}}: \quad$ Demand of customer $k$ for commodity $c$ under scenario $s$.

\subsection{Continuous and Binary Variables}

$X_{i j c s}: \quad$ Amount of commodity $c$ transported from supplier $i$ to distribution center $j$ under scenario $s$,

$Y_{j k c s}: \quad$ Amount of commodity $c$ transported from distribution center $j$ to customer $k$ under scenario $s$,

$\mathrm{I}_{\mathrm{jcs}}$ : Inventory of commodity $c$ which is stored in distribution center $j$ under scenario $s$,

$b_{j k c s}$ : Shortage of commodity $c$ resulting from demand of customer $k$ in distribution center $j$ under scenario $s$,

$b_{\mathrm{kcs}}$ : Shortage of commodity $\mathrm{c}$ for customer $k$ under scenario $s$, which has not been allocated to any distribution center because of limitation and uncertainty of capacity of distribution center,

$\mathrm{n}_{\mathrm{jcs}}$ : $\quad$ Number of orders for commodity $c$ by distribution center $j$ under scenario $s$,

$\theta_{s}: \quad$ The variable applied for linearization of absolute deviation of costs,

$V_{s}: \quad 1$ if the total cost of $S C$ in scenario $s$ is more than a pre-determined amount of budget (BUD) and 0 , otherwise,

$\mathrm{Z}_{\mathrm{j} \mathrm{l}}: \quad \quad \quad \quad \quad$ in case distribution center is opened with size $L$ in location $j$; otherwise, it is 0 .

\subsection{Mathematical Formulation}

In this section, a new robust mathematical model is presented as an extension of Gharegozloo et al. (2013) model, in which uncertainty is stated using a finite number of discrete scenarios. As mentioned before, EOQ approximation approach was applied here to use policy of $(r, Q)$ and determine the number of order of distribution center per year. On the other hand, considering that shortage was permissible but irrecoverable, it was proved that, in the presence of inventory system in this state, economic order was calculated through Wilson relation. Economic order equaled:

$\mathrm{Q}_{j c s}^{*}=\sqrt{\frac{2 \mathrm{D}_{\mathrm{jcs}} \mathrm{F}_{\mathrm{jcs}}}{h_{j c}}}, \quad D_{j c s}=\sum_{k} y_{j k c s}+\sum_{j^{\prime} \neq j} y_{j j^{\prime} c s} \Rightarrow \mathrm{Q}_{j c s}^{*}=\sqrt{\frac{2\left(\sum_{k} y_{j k c s}+\sum_{j^{\prime} \neq j} y_{j j^{\prime} c s}\right) \mathrm{F}_{\mathrm{jcs}}}{h_{j c}}}$

Reordering point was obtained as follows, considering that demand was specified in each scenario:

$r_{j c s}=D_{j c s} \mathrm{LT}=\left(\sum_{k} y_{j k c s}+\sum_{j^{\prime} \neq j} y_{j j^{\prime} c s}\right) \mathrm{LT}$ 
Of course, since the number of ordering was optimized in the model presented in this thesis, economic order can be calculated through the following formula by having optimal number of ordering:

$Q^{*}{ }_{j c s}=\frac{D_{j c s}}{n_{j c s}^{*}}$

In this regard, ordering policy parameters $(r, Q)$ can be calculated. Then, mathematical model of the problem is presented.

$$
\begin{aligned}
& \min \mathrm{Z}_{1}=\sum_{j, l} f_{j l} \mathrm{Z}_{\mathrm{jl}}+\mathrm{TC}_{\mathrm{s}} \\
& \min Z_{2}=\sum_{s \in S} P_{s}\left[T C_{S}-\sum_{s^{\prime} \in S} P_{s^{\prime}} \mathrm{TC}_{s^{\prime}}+2 \theta_{s}\right] \\
& \min Z_{3}=\sum_{s \in S} P_{s} \mathrm{~V}_{s}
\end{aligned}
$$

Eq. (12) is the first objective function, which minimizes the fixed location costs and expected cost of the supply chain. As equation (15) shows, the expected total cost comprises costs of purchase, transportation, inventory holding, shortage in distribution centers, lost sale and ordering along the one-year planning horizon.

$$
\begin{aligned}
& \mathrm{TC}_{S}=\sum_{s \in S} P_{s}\left[\sum_{i, j, c} \phi_{i c s} \mathrm{X}_{\mathrm{ijcs}}+\sum_{i, j, c} C_{i j c s} \mathrm{X}_{\mathrm{ijcs}}+\sum_{j^{\prime} \neq j} \phi_{j^{\prime} c s} \mathrm{y}_{\mathrm{j}^{\prime} \mathrm{jcs}}+\sum_{j, k, c} C_{j k c s} \mathrm{y}_{\mathrm{jkcs}}+\sum_{j \neq j^{\prime}} C_{j j c s} \mathrm{yj}_{\mathrm{jcs}}^{\prime}+\right. \\
& \left.\left.\alpha \sum_{j, c} h_{j c} I_{j c s}+(1-\alpha) \sum_{j, c} h_{j c}^{\prime} I_{j c s}+\sum_{j, k, c} \pi_{k c} \mathrm{~b}_{\mathrm{jkcs}}+\sum_{k, c} \pi_{k c}^{\prime}\left(\mathrm{b}_{\mathrm{kcs}}^{\prime}-\sum_{j} \mathrm{~b}_{\mathrm{jkcs}}\right)+\sum_{j, c} F_{j c} \mathrm{n}_{\mathrm{jcs}}\right)\right]
\end{aligned}
$$

Eq. (13) is the second objective function of this problem, which is variance of purchase, transportation, inventory holding, shortage and ordering costs. This has been considered as absolute magnitude and it is converted to a linear form based on the available literature in this field, like what pointed in the study of Yu and Li (YU \& Li 2000).

Third objective function (14) intends to minimize the probability of not meeting a pre-determined budget or cost level. Where $V_{s}$ is a binary variable and specifies as equation (16):

$V_{s}=\left\{\begin{array}{cc}1 & \text { if } \mathrm{TC}_{\mathrm{s}}>B U D \\ 0 & \text { otherwise }\end{array}\right.$

In other words, the third objective function is the expected number of scenarios in which supply chain total cost violates from the budget restriction. As equation (16) is a nonlinear one, it is linearized by using an additional constraint an arbitrary large number $(\mathrm{M})$ as follows:

$\frac{\mathrm{TC}_{s}-\mathrm{BUD}}{\mathrm{M}} \leq V_{s}<\frac{\mathrm{TC}_{s}}{\mathrm{BUD}}, \forall s$

IfTC $C_{s} \succ B U D$, constraint (17) will be changed to $0 \prec V_{s} \leq 1$ and because $V_{s}$ is a binary variable, it can be deduced $V_{s}=1$.

On the other side, if $T C_{s} \leq B U D$, constraint (17) is altered to $0 \leq V_{s} \prec 1$ which results in $V_{s}=0$. Constraints of the problem are as follows:

Subject to:

$\sum_{i} X_{i j c s}+\sum_{j^{\prime} \neq j} y_{j j c s} \sum_{l} Z_{j l}-\sum_{k} y_{j k c s}-\sum_{j \neq j^{\prime}} y_{j j^{\prime c s}} \sum_{l} Z_{j^{\prime} l}=I_{j c s}-\sum_{k} b_{j k c s} \quad \forall \mathrm{j}, \mathrm{c}, \mathbf{s}$ 


$$
\begin{aligned}
& n_{j c s}=\frac{\left(\sum_{j^{\prime}=j} y_{j j^{\prime} c s}+\sum_{k} y_{j k c s}\right)}{\sqrt{\frac{2\left(\sum_{j^{\prime}=j} y_{j j^{\prime} c s}+\sum_{k} y_{j k c s}\right) \mathrm{F}_{\mathrm{jc}}}{h_{j c}}+\sum_{k} b_{j k c s}}} \forall \mathrm{j}, \mathrm{c}, \mathrm{s},\left(\sum_{j^{\prime}=j} y_{j j^{\prime} c s}+\sum_{k} y_{j k c s}\right)>0 \\
& y_{j j^{\prime} c s} \leq M \sum_{l} Z_{j l} \quad \forall \mathrm{j}, \mathrm{j}^{\prime}\left(\mathrm{j} \neq \mathrm{j}^{\prime}\right), \mathrm{c}, \mathrm{s} \\
& X_{j j c s} \leq M \sum_{l} Z_{\mathrm{jl}} \quad \forall \mathrm{j}^{\prime} \neq \mathrm{j}, \mathrm{c}, \mathrm{s} \\
& \sum_{i} X i j c s \leq M \sum_{l} Z j l \quad \forall \mathrm{j}, \mathrm{c}, \mathrm{s} \\
& \sum_{k} y j k c s \leq M \sum_{l} Z_{j l} \quad \forall \mathrm{j}, \mathrm{c}, \mathrm{s} \\
& \sum_{k, c} v_{c} \mathrm{yjkcs}+\sum_{j^{\prime} \neq j, c} v_{c} \mathrm{y}_{j j}{ }^{\prime} c s \leq \mathrm{q}_{\mathrm{js}} \sum_{l} \operatorname{cap} l \mathrm{Z}_{\mathrm{j} 1} \quad \forall \mathrm{j}, \mathrm{s} \\
& \sum_{j} X_{i j c s} \leq q_{i s} \mathrm{~S}_{\mathrm{ic}} \quad \forall \mathrm{i}, \mathrm{c}, \mathrm{s} \\
& \sum_{l} Z j l \leq 1 \quad \forall \mathrm{j} \\
& \sum_{i} X_{i j c s}-\sum_{j^{\prime} \neq j} y_{j j^{\prime} c s}-\sum_{k} y_{j k c s}<M \alpha \quad \forall \mathrm{j}, \mathrm{c}, \mathrm{s} \\
& \sum_{j^{\prime} \neq j} y_{j j^{\prime} c s}+\sum_{k} y_{j k c s}-\sum_{i} X_{i j c s}<M(1-\alpha) \quad \forall \mathrm{j}, \mathrm{c}, \mathrm{s} \\
& \left\{\begin{array}{ll}
\alpha=1 & \text { if } \sum_{j^{\prime} \neq j} y_{i j^{\prime} c s}+\sum_{k} y_{j k c s}>\sum_{i} X_{i j c s} \\
\alpha=0 & \text { if } \sum_{j^{\prime} \neq j} y_{j j^{\prime} c s}+\sum_{k} y_{j k c s} \leq \sum_{i} X_{i j c s}
\end{array}\right\} \\
& \sum_{j} y_{j k c s}-\sum_{j} b_{j k c s}+b_{k c s}^{\prime}=d_{k c s} \quad \forall \mathrm{k}, \mathrm{c}, \mathrm{s} \\
& Z_{j l} \in\{0,1\} \quad \forall \mathrm{j}, 1 \\
& X_{i j c s} \geq 0, y_{j k c s} \geq 0, I_{j c s} \geq 0, \quad b_{j k c s} \geq 0 \quad \forall \mathrm{i}, \mathrm{j}, \mathrm{c}, \mathrm{s} \\
& \left(\sum_{i, j, c} \phi_{i c s} \mathrm{X}_{\mathrm{ijcs}}+\sum_{j^{\prime} \neq j} \phi_{j^{\prime} c s} \mathrm{y}_{j \mathrm{j} j \mathrm{cs}}+\sum_{i, j, c} C_{i j c s} \mathrm{X}_{\mathrm{ijcs}}+\sum_{j, k, c} C_{j k c s} \mathrm{y}_{\mathrm{jkcs}}+\sum_{j \neq j^{\prime}} C_{j j^{\prime} c s} \mathrm{y}_{j^{\prime} \mathrm{jcs}}+\alpha \sum_{j, c} h_{j c} I_{j c s}+(1-\alpha) \sum_{j, c} h_{j c}^{\prime} I_{j c s}+\right. \\
& \left.\sum_{j, k, c} \pi_{k c} \mathrm{~b}_{\mathrm{jkcs}}+\sum_{k, c} \pi_{k c}^{\prime}\left(\mathrm{b}_{\mathrm{kcs}}^{\prime}-\sum_{j} \mathrm{~b}_{\mathrm{jkcs}}\right)+\sum_{j, c} F_{j c} \mathrm{n}_{\mathrm{jcs}}\right) \\
& -\sum_{s^{\prime} \in S} P_{s^{\prime}}\left(\sum_{i, j, c} \phi_{i c s^{\prime}} \mathrm{X}_{\mathrm{ijcs}}+\sum_{i, j, c} C_{i c c s^{\prime}} \mathrm{X}_{\mathrm{ijcs}}+\sum_{j^{\prime} \neq j} \phi_{j^{\prime} c s^{\prime}} \mathrm{y}_{\mathrm{j}^{\prime} \mathrm{jcs} s^{\prime}}+\sum_{j, k, c} C_{j k c s^{\prime}} \mathrm{y}_{\mathrm{jkcs}}+\sum_{j \neq j^{\prime}} C_{\mathrm{j}^{\prime} j c s^{\prime}} \mathrm{y}_{\mathrm{j}^{\prime} \mathrm{jcs} s^{\prime}}+\right. \\
& \left.\alpha \sum_{j, c} h_{j c} I_{j c s^{\prime}}+(1-\alpha) \sum_{j, c} h_{j c}^{\prime} I_{j c s^{\prime}}+\sum_{j, k, c} \pi_{k c} \mathrm{~b}_{j \mathrm{kcs}}+\sum_{k, c} \pi_{k c}^{\prime}\left(\mathrm{b}_{\mathrm{kcs}}^{\prime}-\sum_{j} \mathrm{~b}_{\mathrm{jkcs}}\right)+\sum_{j, c} F_{j c} \mathrm{n}_{\mathrm{jcs}}\right)+\theta_{s} \geq 0 \quad \forall \mathrm{s} \\
& \theta_{\mathrm{s}} \geq 0 \\
& \forall \mathrm{s} \\
& X_{i j c s}, y_{j k c s}, n_{j c s}, b_{j c s}, Q_{j c s}, r_{j c s} \geq 0 \quad \forall \mathrm{s} \\
& \mathrm{Z}_{\mathrm{j} 1}, \mathrm{~V}_{s} \in\{0,1\}
\end{aligned}
$$

Eq. (18) is the balance equation of inventory in distribution center for each type of commodity. Eq. (19) calculates the number of ordering in the given planning horizon. Constraint (20) shows that 
distribution center $j$ supports other distribution centers as backup center when it has been established with size of $\mathrm{L}$ in location $j$. Constraint (21) also reveals that other distribution centers (j) support distribution center $j$ as a backup center when location $j$ of distribution center with size $\mathrm{L}$ is constructed. Constraints (22) and constraint (23) show supplier $i$ and customer $k$ 's dependency on distribution center $j$. Constraint (24) and (25) show the capacity limitation of distribution center $j$ supplier $i$ considering their active capacity respectively. Constraint (26) enforces that only one distribution center with one possible size can be opened. Constraint (27) depicts that if the commodity amount delivered by suppliers to distribution center $j$ is lower than its demand, it will receive service from backup distribution centers shown using binary parameter of $\alpha$. Constraint (28) is a balance equation for node $\mathrm{k}$ (customer). Constraint (29) is constraint of the problem variables and constraints (30) and (31) are the constraints resulting from linearization of costs variance. Eventually, constraint (32) specifies the type of variables.

\section{Solution procedure}

As the expected total cost, the total cost variance and the financial risk are in contrast with each other(Azaron et al., 2008), here a multi-objective mathematical model is proposed based on Epsilon Constraint Method and Lingo 9 software is implemented to create a set of Pareto optimal possible solution. Epsilon Constraint Method is one of the common approaches for dealing with multiobjective problems, which solves such problems by considering all objective functions as constraints and retaining only one of them in each phase as the main objective function (Ehrgott \& Gandibleux, 2002). In this case, Pareto Border can be constructed by $\varepsilon$ constraint method (Bérubé et al. 2009).

$$
\min f_{1}(x) \mid x \in X, f_{2}(x) \leq \varepsilon_{2}, \cdots, f_{n}(x) \leq \varepsilon_{n}
$$

The algorithm of $\varepsilon$-constraint method defines as follows:

1- Select one of the objective functions is selected as the main objective function,

2- For each objective functions, solve the problem and find the optimal values of each objective function,

3- To specify the amount of $\varepsilon_{2}, \ldots, \varepsilon_{\mathrm{n}}$, the interval between two optimal values of objective subfunctions is divided into $n(\beta)$ and then by a recursive equation the $\varepsilon_{2}, \ldots, \varepsilon_{n}$ will be calculated.

4- Solve, at any time, with main objective function with each value of $\varepsilon_{2}, \ldots, \varepsilon_{n}$.

5- Repeat the proposes until Pareto's solutions are detected.

To verify the proposed model as a three-objective one, a test problem is studied with the problem codes of 2-1-3-2-3. 10 sub-problems are conducted in whichz $z_{2}$ 's optimal value is determined at any time by selecting the second objective function as the main objective function and setting the first objective function in constraint based on $\varepsilon_{2}, \ldots, \varepsilon_{\mathrm{n}}$. The results of these steps are shown in Table 2 .

$$
Z_{1}-Z_{1}^{*}=D, \quad \beta=D / n, \quad \varepsilon_{n}=\varepsilon_{n-1}+\beta
$$

\section{Numerical Examples}

Here, as the model is NP-hard due to a long run time and existing literature, we use two example of small sizes, which lead to global solutions based on each objective function. To solve these problems, Epsilon Constraint method, which is described in Section 4.1, is used in lingo9 software. all the tests are implemented on Pentium3 (Intel (R) Core ${ }^{\mathrm{TM}}$ 2CPUT5500@1.66GHz) and Microsoft Windows XP Professional operating system. Then, data of these problems are presented in Table 1. In these numerical examples we consider a single type of commodity for the sake of clarity in the representation. As the number of grid points enlarges, the Pareto optimal solution set increases accordingly. The number of grid points for each objective function could be determined based on the range of that objective function, i.e. in the case of financial risk, which varies from 0 to 1; it could be divided to 3 or 9 grid points according to the DM expectation. Using the Branch and bound method the ideal and the nadir values for each objective functions are obtained and reported in Table 2. 


\section{Table 1}

Data of numerical examples

\begin{tabular}{cccc}
\hline Parameter & Characteristic & Parameter & Characteristic \\
\hline $\mathrm{C}_{\mathrm{ijcs}}(\$ /$ unit-km) & $\mathrm{U}(0.05,0.15)$ & $\mathrm{cap}_{\mathrm{l}}\left(10^{3} \mathrm{~m}^{3}\right)$ & $(100,150,300)$ \\
$\mathrm{C}_{\mathrm{i}{ }^{\prime} \mathrm{css}}(\$ /$ unit-km) & $\mathrm{U}(0.05,0.15)$ & $\mathrm{f}_{\mathrm{jl}}\left(10^{3} \$\right)$ & $(6,10,16)$ \\
$\mathrm{C}_{\mathrm{ikcs}}(\$ /$ unit-km) & $\mathrm{U}(0.05,0.15)$ & $\mathrm{d}_{\mathrm{kcs}}\left(10^{3}\right.$ unit $)$ & $\mathrm{U}(50,100)$ \\
$\mathrm{P}_{\mathrm{s}}\left(\sum \mathrm{P}_{\mathrm{s}}=1\right)$ & $\mathrm{U}(0,1)$ & $\mathrm{S}_{\mathrm{ic}}\left(10^{3}\right.$ unit $)$ & $\mathrm{U}(0.5,1)$ \\
$\varphi(\$)_{\mathrm{ics}}$ & $\mathrm{U}(200,210)$ & $\mathrm{q}_{\mathrm{is}}$ & $\mathrm{U}(0.5,1)$ \\
$\varphi_{\mathrm{j} \mathrm{j}^{\prime} \mathrm{cs}}(\$)$ & $\mathrm{U}(205,215)$ & $\mathrm{q}_{\mathrm{js}}$ & 1000 \\
$v_{\mathrm{c}}\left(\mathrm{m}^{3}\right)$ & $2-3$ & $\mathrm{M}$ & 250 \\
$\mathrm{~h}_{\mathrm{jc}}(\$)$ & 200 & $\mathrm{~F}_{\mathrm{jc}}(\$)$ & $2000(10$ times bigger than minimum procurement cost $)$ \\
$\mathrm{h}_{\mathrm{ic}}(\$)$ & 205 & $\pi_{\mathrm{kc}}(\$)$ & $2500(12.5$ times bigger than minimum procurement cost $)$ \\
\hline
\end{tabular}

Table 2

$\varepsilon$-constraint results in lingo 9

\begin{tabular}{|c|c|c|c|c|c|c|c|}
\hline $\mathrm{P}$ & S/SUP/DC/COM./CUS. & The optimal solution for $\mathrm{i}$-th single objective $\mathrm{m}$ & $Z_{1}(x)$ & $Z_{2}(x)$ & $Z_{3}(x)$ & CPU & \\
\hline & \multirow{4}{*}{$2 / 2 / 2 / 1 / 2$} & $x_{1}^{*}$ & 32103.84 & 34886.2 & 0.2 & 42 & \multirow{4}{*}{ 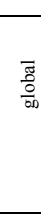 } \\
\hline \multirow[t]{2}{*}{ 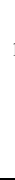 } & & $x_{2}^{*}$ & 53382 & 25216.1 & 0.8 & 71 & \\
\hline & & $x_{3}{ }^{*}$ & 52695 & 34526.4 & 0 & 112 & \\
\hline & & $\begin{array}{l}\text { Ideal value } \\
\text { Nadir value }\end{array}$ & 32103.85 & 25216.1 & $\begin{array}{c}0 \\
0.8 \\
\end{array}$ & & \\
\hline \multirow{4}{*}{2} & \multirow{4}{*}{$3 / 2 / 2 / 1 / 2$} & $x_{1}^{*}$ & 53097.26 & 32246.48 & 0.65 & 67 & \multirow{4}{*}{$\frac{\mathrm{og}}{60}$} \\
\hline & & $x_{2}{ }^{*}$ & 56989.1 & 26796.58 & 0.85 & 72 & \\
\hline & & $x_{3}{ }^{*}$ & 88400.6 & 56946.22 & 0 & 38 & \\
\hline & & $\begin{array}{l}\text { Ideal value } \\
\text { Nadir value }\end{array}$ & 53097.3 & 26796.6 & $\begin{array}{c}0 \\
0.85\end{array}$ & & \\
\hline
\end{tabular}

We consider the first objective function (total cost of supply chain) as the main objective function and divide the other objectives' ranges 4 grid points for both variability and financial risk. As we can observe from the results of Table4, for small instances, global solutions are available as the model is NP-hard. Therefore, we just focus on problem resulting in global solution.
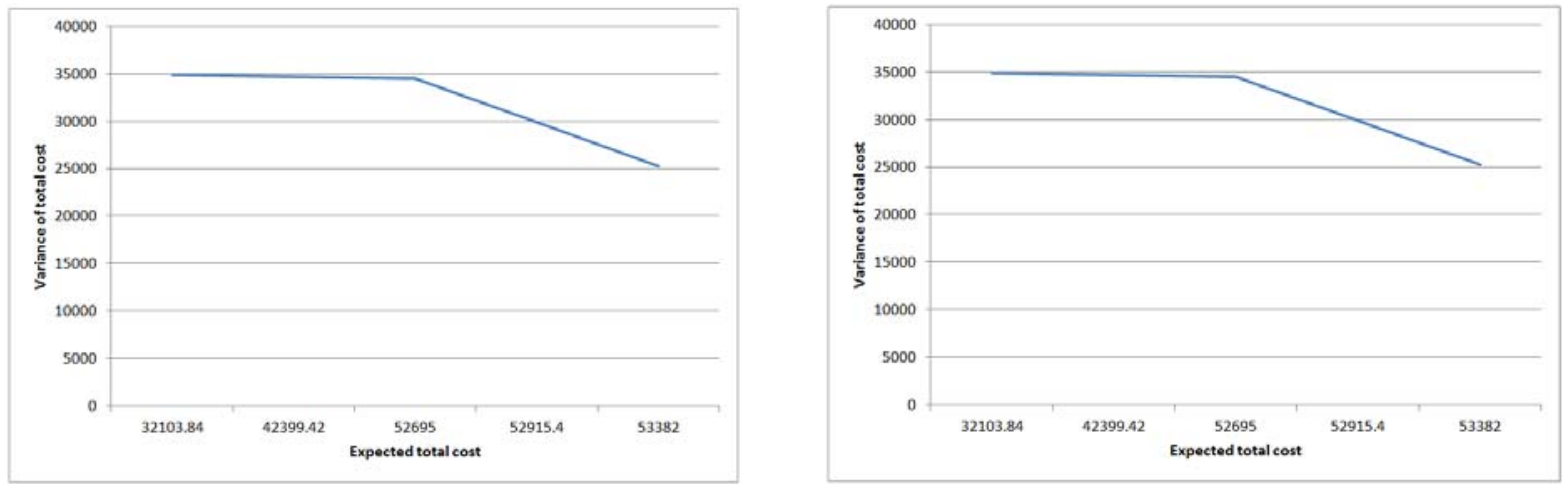

Fig.1. Efficient frontier for test problem 1
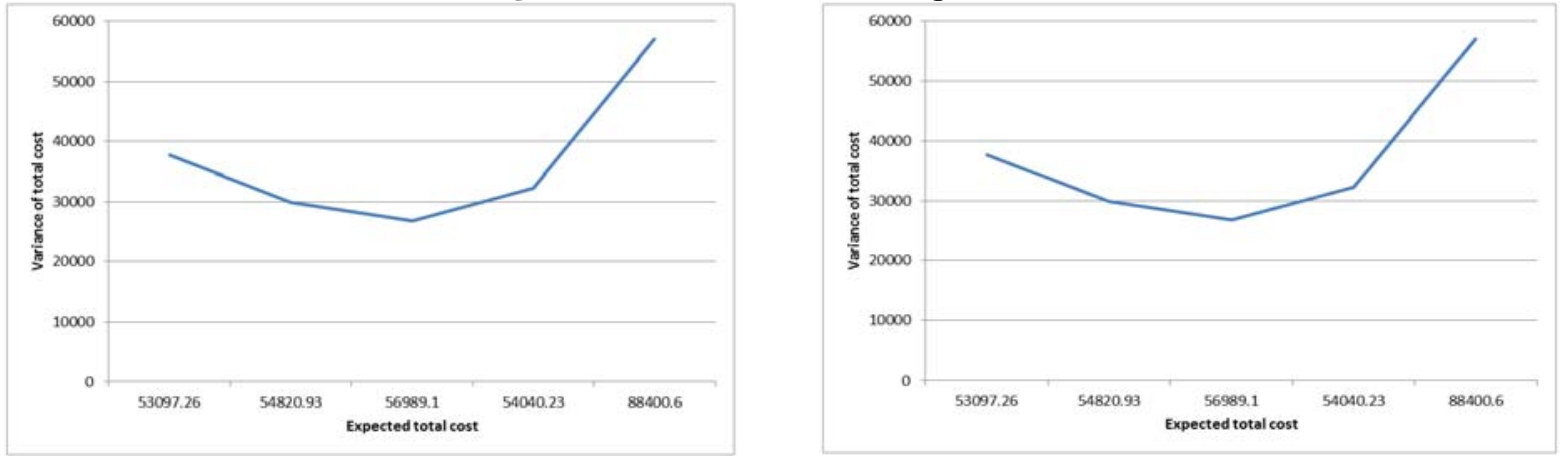

Fig. 2. Efficient frontier for test problem 2 
We consider the first objective function (total cost of supply chain) as the main objective function and divide the other objectives' ranges by 4 grid points for both variability and financial risk. The efficient frontiers are given in Fig. 1 and Fig. 2 for problem 1 and 2, respectively. As can be seen, generally, there is a considerable conflict between the expected value and the variability of TC of the given supply chain as well as the expected value and the financial risk in both problem 1 and 2 . This condition is due to this fact that in the case of expected total cost, the model tries to find the solutions that they have a good expected value not regarding to the variability of the total cost under realization of the different scenarios.

Conversely, in the case of the variability of total cost, the model tries to find solutions that they have objective values as close as possible to each other under realization of the different scenarios not regarding to the objective values.

\section{Conclusion and future research}

In this paper a multi objective stochastic programming model has been developed to deal with uncertain three-level supply chain considering risk and inventory decisions where two more objective functions including variability and financial risk has beenadded and a mathematical method has been applied to linearize the proposed model. In the model, the cost parameters of the supply chian as well as demand have been exposed to uncertainty. The proposed model has been solved with the $\varepsilon$ constraint method. A numerical example is randomly generated and the results exhibited the validity and the efficiency of the proposed method. This work can be developed in some directions: first, the proposed model can be aggregated with the other uncertain factors like lead times. Second, for largescale problems, especially those involving a simultaneous increase in the number of scenarios, the number of commodities and the number of DCs, it would be necessary to apply meta-heuristics. Finally, considering the model for more than three level supply chains and routing decisions can be considered as promising contribution and we leave it for interested researchers as future studies.

\section{References}

Alvarez-Benitez, J. E., Everson, R. M., Fieldsend, J.E.. (2005). A MOPSO Algorithm Based Exclusively on Pareto Dominance Concepts. Springer-Verlag Berlin Heidelberg, 459-473.

Axsater, S. (2006). inventory control, Second edition, Springer, New York.

Azaron, A., Brown, K. N., Tarim, S.A., \& Modarres, M. (2008). A multi-objective stochastic programming approach for supply chain design considering risk. international Journal of Production Economics, 116, 129-138.

Berman, O., Krass, D., \& Tajbakhshm, M. M. (2012). A coordinated location-inventory model. European Journal of Operational Research, 217, 500-508.

Bérubé, J. F., Gendreau, M., \& Potvin, J.Y. (2009). An exact $\varepsilon$-constraint method for bi-objective combinatorial optimization problems: Application to the traveling salesman problem with profits. European Journal of Operational Research, 194, 39-50.

Bozorgi-Amiri, A., Jabalameli, M. S., Alinaghian, M., \& Heydari, M. (2012). A modified particle swarm optimization for disaster relief logistics under uncertain environment. International Journal of Advance Manufacturing Technology, 60, 357-371.

Chen, Q., Li, X., \& Ouyang, Y. (2011). Joint inventory-location problem under the risk of probabilistic facility disruptions. Transportation Research Part B, 45,991-1003.

Daskin, M. S., Coullard, C. R. , \& Shen, Z.-J.M. (2002). An inventory-location model: Formulation, solution algorithm and computational results. Annals of Operations Research, 110, 83-106.

Eckart, Z., Kalyanmoy, D., \& Lothar, T. et al. (2000). Comparison of Multi objectiveEvolutionary Algorithms: Empirical Results. Evolutionary Computation, 8(2), 173-195.

Ehrgott, M., \& Gandibleux, X. (2002). Multi objective combinatorial optimization theory, methodology and applications. Kluwer Academic. 
Eppen , G. D. (1979). Effects of centralization on expected costs in a multi-location newsboy problem.Management Science, 25, 498-501.

Erlebacher, S., \& Meller, R. (2000). The interaction of location and inventory in designing distribution systems. international industrial engineering Transactions, 32, 155-166.

Gharegozloo Hamedani, S., Jabalameli, M.S., \& Bozorgi-Amiri, A. (2013). A location-inventory model for distribution centers in a three-level supply chain under uncertainty. International Journal of industrial engineering computations, 4(1), 93-110.

Kennedy, J., \& Eberhart, R. C. (2001). Swarm Intelligence. San Francisco, California, Morgan Kaufmann Publishers.

Megiddo, N., \& K. Supowit (1984). On the complexity of some common geometric location problems. society for industrial and applied mathematics journal on computing, 13, 182-196.

Melo, M., Nickel, S., \& Saldanha-da-Gama, F. (2009). Facility location and supply chain management - a review. European Journal of Operational Research, 196(2), 401-412.

Min, H., \& Zhou, G. (2002). Supply chain modeling: past, present and future. Computer and Industrial Engineering, 43, 231-249.

Mulvey, J. M., Vanderbei, R. J., \& Zenios, S. A. (1995). Robust optimization of large scale systems. operational Research, 43(2), 264-281.

Nozick, L., \& Turnquist, M. (1998). Integrating inventory impacts into a fixed-charge model for locating distribution centers. Transportation Research Part E, 34(3), 173-186.

Park, S., Lee, T., \& Sung, C. (2010). A three-level supply chain network design model with riskpooling and lead times. Transportation Research Part E, 46, 563-581.

Qi, L., Shen, Z. J., \& Snyder, L.V. (2010). The effect of supply disruptions on supply chain design decisions. Transportation Science, 44(2), 274-289.

Rawls CG, Trunquist MA (2010) Pre-positioning of emergency supplies for disaster response. Transportation research part B: Methodological 44(4): 521-534.

Romeijn, H. E., Shu, J., \& Teo, C. (2007). Designing two-echelon supply networks. European Journal of Operational Research, 178, 449-462.

shen, Z. J., Coullard, C., \& Daskin, M.S. (2003). A joint location-inventory model. transportation science, 37, 40-55.

Shen, Z. J., \& Qi, L. (2007). Incorporating inventory and routing costs in strategic location models. European Journal of Operational Research, 179, 372-389.

Snyder, L. V. (2006). Facility location under uncertainty: A review. international Industrial Engineering Transactions, 38(7), 547-564.

Snyder, L. V., Daskin, M. S., \& Teo, C. ( 2007). The stochastic location model with risk pooling. European Journal of Operational Research, 179(3), 1221-1238.

Sourirajan, K., Oszen, L., \& Uzsoy, R. (2007). A single product network design with leadtime and safety stock consideration. HE Transaction,39(5), 411-424.

Tancrez, J.-S., Lange , J.-C. ,\& Semal, P. (2012). A location-inventory model for large three-level supply chains. Transportation Research Part E, 48, 485-502.

Teo, C., \& Shu, J. (2004). Warehouse-retailer network design problem. Operations Research, 52, 396-408.

Tsao, Y.-C., Mangotra, D., Lu, J.C., \& Dong, M. (2012). A continuous approximation approach for the integrated facility-inventory allocation problem. European Journal of Operational Research. 222,(216-226).

YU, C., \& Li, H. (2000). a robust optimization model for stochastic logistic problems. international Journal of Production Economics, 64(1-3), 385-397.

Üster, H., Keskin, B., \& Çetinkaya, S. (2008). Integrated warehouse location and inventory decisions in a three-tier distribution system. International Industrial Engineering, 40, 718-732. 